\title{
MODALIDADES DE COOPERACIÓN INTERNACIONAL UNIVERSITARIA EN EL CONTEXTO DE LA INTERNACIONALIZACIÓN DE LA EDUCACIÓN SUPERIOR ARGENTINA
}

\section{UNIVERSITY INTERNATIONAL COOPERATION TYPES FOR THE INTERNATIONALIZATION OF ARGENTINEAN HIGHER EDUCATION}

\author{
Gabriela Michelini \\ Fernando Luján Acosta \\ Mónica Vivian Ettlin
}

\section{RESUMEN}

El presente artículo analiza los aspectos jurídicos - institucionales de la cooperación internacional universitaria con el objetivo de presentar las modalidades que puede asumir en el entorno iberoamericano, desde el análisis del caso argentino. La metodología aplicada fue el análisis sistemático de documentos normativos nacionales y recomendaciones de organismos internacionales. Los resultados principales destacan la centralidad del rol del Estado en la promoción de entornos específicos de cooperación universitaria. Asimismo, se observa que las modalidades de cooperación internacional universitaria se corresponden con las funciones universitarias de docencia, investigación y extensión.

\section{PALABRAS CLAVE}

Internacionalización de la educación superior, cooperación internacional descentralizada, cooperación internacional universitaria

\begin{abstract}
This paper analyses the juridical and institutional issues of international cooperation among universities. The aim is to introduce types of university cooperation in the Ibero-American context, from the perspective of the internationalization of Argentinean universities. The methodology used was a systematic analysis of national and international documents. The main results highlight the role of State in promoting cooperation and the correspondence between university international cooperation types and the university three-fold functions: teaching, research and extension.
\end{abstract}

\section{KEY WORDS}

Internationalization of higher education, international decentralised cooperation, international university cooperation 
Gabriela Michelini

Fernando Luján Acosta

Mónica Vivian Ettlin

Modalidades de cooperación internacional universitaria en el contexto de la internacionalización de la educación superior argentina Págs. 86-115

\section{INTRODUCCIÓN}

La estrecha relación entre educación y desarrollo ha sido objeto de múltiples debates y definición de agendas estratégicas a nivel mundial. La Declaración de la Conferencia Mundial sobre Educación Superior de 2009 postula que "la educación superior y la investigación contribuyen a la erradicación de la pobreza, al desarrollo sustentable y al progreso en el alcance de las metas de desarrollo acordadas en el ámbito internacional" (UNESCO, 2009: s/p). Esta definición se enmarca en la consolidación de la economía del conocimiento, que define tanto una forma de la producción capitalista, basada en la capacidad de generar valor a partir del la aplicación de saberes técnicos y científicos, como un ámbito de estudio de la desigualdad en ese entorno (Vessuri, 2008). Así, la producción tecnológica y de conocimiento en el contexto actual es parte de la ecuación de poder de los Estados en el sistema mundial ya que puede ser considerada como un elemento clave para el fortalecimiento de las capacidades militares, económicas y simbólicas de los Estados (Altbach, 2009).

En este contexto, las universidades se han convertido en "instituciones del mundo global, además de asumir sus roles tradicionales locales y nacionales" (Aebischer, 2015: 3). La transformación de la universidad no ha sido un proceso inmediato ni aislado. Su retórica mutó de una noción asistencialista de la dimensión internacional, para la mejora de las capacidades de los países con menor desarrollo relativo, hasta la asimilación de la dimensión internacional como elemento constitutivo de las políticas de educación superior (De Witt; 2010).

Este avance hacia lo internacional en la educación superior se desarrolla en formas complejas a través de la armonización de normas, estandarización de procesos, mayor difusión de la información y la imposición de criterios y políticas (Rama, 2015). En este sentido, la internacionalización de la educación superior constituye el proceso de integrar las dimensiones internacionales, interculturales y globales en el objetivo, función y provisión de la educación superior (Knight, 2005). La definición del fenómeno implica, por lo tanto, la incorporación de tendencias que exceden a lo meramente educativo, pero que insertan dentro del ámbito universitario nuevas prácticas, tensiones y definiciones que reorientan la definición misma de la actividad universitaria. 
Gabriela Michelini Fernando Luján Acosta Mónica Vivian Ettlin Modalidades de cooperación internacional universitaria en el contexto de la internacionalización de la educación superior argentina Págs. 86-115

Dentro de las implicancias del proceso de internacionalización, se encuentra una de las acciones específicas en las que se puede observar y medir el fenómeno en las universidades: la cooperación universitaria internacional. Este trabajo se propone explorar los aspectos jurídicos - institucionales de la cooperación universitaria internacional en Argentina, con el objetivo de presentar un estado de la cuestión que considere características, modalidades e interacciones con las distintas funciones de la universidad como institución. Con este fin, se realizó una revisión bibliográfica sobre los conceptos de internacionalización de la educación superior y la cooperación internacional universitaria. Luego, se realizó un análisis sistemático de normativa a nivel nacional y de documentos internacionales que abordan las acciones de cooperación internacional universitaria. En los próximos párrafos, se introduce una propuesta de abordaje de la cooperación internacional universitaria como parte del proceso de internacionalización.

\section{LA INTERNACIONALIZACIÓN DE LA EDUCACIÓN SUPERIOR Y LA FUNCIÓN DE LA UNIVERSIDAD EN LA PRODUCCIÓN DE CONOCIMIENTO}

Aunque se puede trazar el componente internacional de las universidades ya en su origen medieval, caracterizado por la movilidad de estudiantes y académicos en la construcción de un saber universal (Haug, 2010); las instituciones universitarias tal como llegan al día de hoy, se relacionan más bien con una institución de conocimiento cuyo modelo fue central para la construcción del Estado-Nación (Wittrock, 1993). Las funciones y actividades de la universidad se dan en el marco de sistemas o ámbitos nacionales de educación superior. $Y$ en este contexto, la literatura revisada en el tema describe a las universidades como sujetos y objetos de un proceso de internacionalización (Enders, 2004).

En este sentido, los autores coinciden en vincular la internacionalización de la educación superior con un proceso dinamizado en los 80 (Sebastián, 2004; Knight, 2005), que asume características distintas de otras formas anteriores de relacionamiento internacional de la universidad (Perrotta, 2016)

En América Latina, el fortalecimiento de las estrategias de internacionalización de la educación superior se registró de la mano de la inserción de los Estados en la economía global, con la intervención de organismos internacionales y el 
Gabriela Michelini Fernando Luján Acosta

Mónica Vivian Ettlin

Modalidades de cooperación internacional universitaria en el contexto de la internacionalización de la educación superior argentina Págs. 86-115

establecimiento de políticas de promoción de la internacionalización tanto a nivel institucional como gubernamental (Gacel-Ávila, 2005; Didou Aupetit, 2014). Los criterios utilizados se basaron en la literatura norteamericana sobre el tema y la experiencia europea en el proceso de Bolonia (Stubrin, 2011; Oregioni, 2015). No obstante, a comienzos del Siglo XXI el fenómeno de la internacionalización se convirtió en objeto de numerosos estudios latinoamericanos que tienen como característica común un marcado enfoque nacional (Didou Aupetit, 2014).

Cabe señalar, en este punto, que la universidad en América Latina tiene un rol central en la producción de conocimiento, ya que es tanto el ámbito de desarrollo de la investigación como el de formación de profesionales y académicos, como lo indican los datos publicados por la Red de Indicadores de Ciencia y Tecnología (RICYT), el sector de empleo predominante de los investigadores en Argentina es la Educación Superior, donde se encuentran el $56,4 \%$ del total de investigadores (RICYT, 2015).

La Ley de Educación Superior № 24.521 (LES) de Argentina es el marco regulatorio que introduce la evaluación y el aseguramiento de la calidad como eje de la política universitaria (CONEAU, 2012), al mismo tiempo que explicita triple función de la universidad en su Art. 28, en el que indica que es función de la universidad:

a) Formar y capacitar científicos, profesionales, docentes y técnicos, capaces de actuar con solidez profesional, responsabilidad, espíritu crítico y reflexivo, mentalidad creadora, sentido ético y sensibilidad social, atendiendo a las demandas individuales, en particular de las personas con discapacidad, desventaja o marginalidad, y a los requerimientos nacionales y regionales. (Esta versión corresponde al inciso sustituido por art. $3^{\circ}$ de la Ley $N^{\circ} 25.573$ publicada en el B.O. el 30/04/2002)

b) Promover y desarrollar la investigación científica y tecnología, los estudios humanísticos y las creaciones artísticas;

c) Crear y difundir el conocimiento y la cultura en todas sus formas;

d) Preservar la cultura nacional;

e) Extender su acción y sus servicios a la comunidad, con el fin de contribuir a su desarrollo y transformación, estudiando en particular los problemas nacionales y regionales y prestando asistencia científica y técnica al Estado y a la comunidad. (Argentina, Ley $\left.\mathrm{N}^{\circ} 24.521,1995\right)$.

En las funciones de la universidad descansan sus tres acciones centrales respecto del 
Gabriela Michelini Fernando Luján Acosta

Mónica Vivian Ettlin

Modalidades de cooperación internacional universitaria en el contexto de la internacionalización de la educación superior argentina

Págs. 86-115

conocimiento: producción, formación y difusión. El imperativo de la norma llevó al dinamismo del área de investigación que hasta entonces no estaba necesariamente desarrollada en las universidades (Barsky et. al., 2016). Este dinamismo se vincula con otra innovación (Sánchez Martínez, 2002): la LES establece lo relativo a la evaluación institucional y acreditación de carreras. De este modo, pauta la obligación para todas las instituciones universitarias, estatales y privadas, de someterse regularmente a procesos de evaluación y determina en su artículo 43 que las carreras de interés público deberán someterse a acreditación.

La evaluación y la acreditación constituyen herramientas para una doble finalidad. Por un lado, permiten cierta homogeneización y articulación interna del sistema. Por el otro, esa misma homogeneización es la que sienta las bases para la proyección internacional de las actividades realizadas por las universidades. De este modo, la evaluación y la acreditación impactan en el cambio institucional (Corengia, 2015). Cabe suponer, entonces, que las acciones que emprenden las universidades en el marco de su internacionalización (como la cooperación internacional), tienen relevancia en los procesos de cambio institucional y en la forma en la que las universidades desarrollan sus funciones.

\subsection{Precisiones conceptuales sobre la internacionalización como proceso}

La definición más difundida de la internacionalización de la educación superior (Beneitone, 2014) establece que se trata del proceso de integrar las dimensiones internacionales, interculturales y globales en el objetivo, función y provisión de la educación superior (Knight, 2005).

El proceso referido se presenta en tres dimensiones relativas a la incorporación de lo internacional: 1) movilidad de estudiantes y docentes; 2) la mercantilización de la educación superior, a partir de la difusión de un modelo competitivo, el concepto de educación como servicio y la instalación de universidades extranjeras y de empresas proveedoras de educación superior; y 3) la virtualización y la educación a distancia (Dávila, 2008; Altbach, 2009; Didou Aupetit, 2009). Otras tipologías suman además las siguientes dimensiones: 4) la consolidación de sistemas internacionales de evaluación y acreditación universitaria; 5) la internacionalización del currículo; 6) la formación de profesionales para un mercado laboral global; 7) la difusión de un modelo de 
Gabriela Michelini Fernando Luján Acosta

Mónica Vivian Ettlin

Modalidades de cooperación internacional universitaria en el contexto de la internacionalización de la educación superior argentina Págs. 86-115

universidad centrado en la productividad y en los rankings internacionales; 8) la consolidación del inglés como lengua de circulación del conocimiento; 9) la proliferación del uso de Internet para la docencia y la investigación; 10) las creación de redes y asociaciones de universidades (Rama, 2015; Perrotta, 2016).

Como es posible observar, esta tipificación de la internacionalización de la educación superior refiere más bien a las dimensiones que en las que la internacionalización se expresa, antes que a las características específicas del proceso. La internacionalización resulta así un proceso integrador y no un mero grupo de actividades aisladas (Miranda, 2008: 8).

De acuerdo a la orientación del proceso de internacionalización, se diferencian dos modelos predominantes. El modelo solidario, orientado al entendimiento y el relacionamiento horizontal y el modelo competitivo o fenicio, caracterizado por la orientación al mercado (Perrotta, 2016). En este sentido, Azevedo (2015) presenta una diferenciación conceptual al reservar el modelo competitivo al fenómeno de transnacionalización de la educación superior, esto es, la conformación de un mercado global de educación superior; y el modelo del relacionamiento horizontal a un ámbito global en el que la educación es compartida como un bien social, de acuerdo a lo estipulado por la Conferencia Regional de Educación del 2008 (CRES).

Este artículo se centra en las modalidades de cooperación internacional universitaria como parte de la internacionalización en este segundo sentido conceptual, como ámbito global de acción solidaria, y por lo tanto, se soslayan en el análisis los impactos que tiene en este sentido la mercantilización de la educación superior.

De este modo, se observa también que la internacionalización remite a un proceso más amplio, del cual la cooperación es parte. La internacionalización refiere a un proceso por el cual la universidad se presenta como un actor que busca ventajas en su interrelación con otros actores del ámbito internacional, mientras que la cooperación internacional universitaria refiere a una de las acciones posibles de esa interrelación entre actores pares (Beneitone, 2014).

\section{2. La cooperación internacional}


Gabriela Michelini

Fernando Luján Acosta

Mónica Vivian Ettlin

Modalidades de cooperación internacional universitaria en el contexto de la internacionalización de la educación superior argentina

Págs. 86-115

La cooperación internacional refiere a la vinculación entre países que persiguen un beneficio o resultado común como un acto de corresponsabilidad o de "asociados" (Miranda, 2008: 7; Siufi, G, 2009: 124). Las miradas más tradicionales de la cooperación, vinculadas al paradigma clásico "asistencialista" o "verticalista" o de la escuela realista de la política internacional, centran este proceso en las acciones de los Estados- nación. Es posible denominar a este proceso cooperación internacional centralizada (Socas y Hourcade, 2009). Sus actores son los Estados y las instituciones por ellos creadas. Sus modalidades son:

a) Modalidad bilateral, entre dos Estados: Cooperación Norte - Sur: si se da entre un país desarrollado y un país receptor de la ayuda no desarrollado; Cooperación SurSur: si se da entre dos países no desarrollados.

b) Modalidad trilateral, entre tres Estados: generalmente adopta se conforma entre un país desarrollado con otros dos no desarrollados.

c) Modalidad multilateral: varios Estados desarrollados y no desarrollados; incluimos aquí la cooperación brindada por organismos internacionales gubernamentales multilaterales y el país o países que requieren la cooperación internacional.

Esto no quiere decir, no obstante, que cooperación equipare a la armonía en el funcionamiento del sistema internacional. La cooperación sucede porque cuando cada país persigue su propio interés puede encontrar otros países con intereses convergentes, de modo tal que la asociación facilita el cumplimiento de los objetivos.

Sin embargo, en este contexto, se debe considerar los países siempre se verán limitados por "las preferencias de los actores más poderosos" de modo tal que el contexto estructural siempre impactará en las decisiones de los actores (Keohane, 1985: 99).

Russell y Tokatlian plantean el concepto de "esfera de cooperación" que supone que "es posible crear ámbitos de colaboración en contextos de gran disparidad de poder y competencia y que la cooperación es producto de la elección y de la circunstancia" (2005: 92). Sus funciones son: "proporcionar estabilidad, gestionar conflictos; conjurar crisis; difundir poder; dotar de mayor capacidad de acción a los actores menores; generar confianza; reducir incertidumbre; y evitar fallas de percepción" (Russell y 
Gabriela Michelini Fernando Luján Acosta

Mónica Vivian Ettlin

Modalidades de cooperación internacional universitaria en el contexto de la internacionalización de la educación superior argentina Págs. 86-115

Tokatlian; 2005: 94).

La globalización ha traído desafíos que requieren de la cooperación, el acuerdo y la gestión conjunta en el plano internacional bajo formas de interacción que multiplican los actores de la cooperación internacional. Como señala Paramio, queda implícito en este razonamiento que "el proceso de globalización ha arrebatado a los gobiernos nacionales gran parte de su capacidad de decisión y actuación, que ha pasado a manos de los mercados financieros, las instancias multilaterales o los gobiernos locales subnacionales" (Paramio, 2003: 3).

En este marco, los actores de la cooperación ya no son únicamente los estados nacionales y los organismos internacionales por estos creados, sino también los gobiernos subestatales (provincias, departamentos, municipios, etc.), diferentes organismos autónomos y autárquicos, como las universidades públicas y los centros de investigación, las empresas y también otras organizaciones de la sociedad civil y del sector privado. En este ámbito aparece la categoría de cooperación internacional descentralizada, que contempla las acciones de cooperación llevadas a cabo por los entes subestatales, donde conviven modos de cooperación internacional estatal y no estatal, sobre la base del paradigma de gobernanza multinivel (Socas y Hourcade, 2009). A ellos se suma la creciente conformación de modos asociativos formales y de redes de vinculación informales. De este modo, es posible establecer una tipología de cooperación internacional de acuerdo a lo que se especifica en el Cuadro $\mathrm{N}^{\circ} 1$.

En tanto, una de las formas tradicionales que asume la cooperación internacional universitaria en el contexto latinoamericano es la que fue agrupada como procesos de cooperación Sur - Sur, concepto acuñado en los 70 por los países del Sur global para redefinir reforzar su capacidad de negociación con el Norte, refiriendo a las acciones cooperativas en relación al comercio, el desarrollo y el nuevo orden económico internacional (Lechini, 2007). 
Gabriela Michelini

Fernando Luján Acosta

Mónica Vivian Ettlin

Modalidades de cooperación internacional universitaria en el contexto de la internacionalización de la educación superior argentina

Págs. 86-115

Cuadro № 1. Tipología de cooperación internacional

\begin{tabular}{|c|c|c|c|}
\hline Características & \multicolumn{3}{|c|}{ Cooperación internacional } \\
\hline Tipo & \multicolumn{2}{|c|}{ Estatal } & No estatal \\
\hline Categoría & Centralizada & Centralizada Indirecta & Descentralizada \\
\hline $\begin{array}{l}\text { Actores } \\
\text { participantes }\end{array}$ & $\begin{array}{l}\text { Estados Nacionales } \\
\text { Organismos } \\
\text { Internacionales } \\
\text { Gubernamentales } \\
\text { Agencias Estatales de } \\
\text { cooperación } \\
\text { internacional }\end{array}$ & $\begin{array}{ll}\text { Estados provinciales } \\
\text { Regiones } & \\
\text { Municipios } & \\
\text { Universidades } & \text { de } \\
\text { gestión estatal } & \\
\text { Centros } & \text { de } \\
\text { investigación } & \text { de } \\
\text { gestión estatal } & \end{array}$ & $\begin{array}{lr}\text { Organizaciones } & \text { No } \\
\text { Gubernamentales } & \\
\text { Asociaciones } & \\
\text { Fundaciones } & \\
\text { Universidades } & \text { privadas } \\
\text { Sindicatos } & \\
\text { Organizaciones } & \text { de } \\
\text { carácter social } & \\
\text { Centros } & \text { de } \\
\text { investigación } & \text { de } \\
\text { carácter privado } & \\
\text { Sector privado } & \\
\text { Empresas } & \\
\text { Entidades } & \\
\text { privadas } & \\
\end{array}$ \\
\hline Modalidades & $\begin{array}{l}\text { 1. Bilateral: entre dos } \\
\text { Estados. } \\
\text { a) País donante } \\
\text { desarrollado - país } \\
\text { receptor de la ayuda: } \\
\text { Cooperación: NORTE- } \\
\text { SUR } \\
\text { b) Cooperación: SUR - } \\
\text { SUR horizontal (países } \\
\text { cooperantes) } \\
\text { 2. Trilateral: un país } \\
\text { desarrollado con otros } \\
\text { en vía de desarrollo. } \\
\text { 3. Multilateral: entre } \\
\text { varios países } \\
\text { desarrollados y/o en vías } \\
\text { de desarrollo }\end{array}$ & $\begin{array}{l}\text { 1. Acuerdos de } \\
\text { cooperación de entes } \\
\text { subestatales, sobre la } \\
\text { base de la autonomía } \\
\text { de dichos agentes: } \\
\text { Provincias, } \\
\text { Municipios, regiones, } \\
\text { Ciudades Autónomas, } \\
\text { Entidades } \\
\text { Autárquicas, } \\
\text { Universidades } \\
\text { Públicas, etc. }\end{array}$ & $\begin{array}{l}\text { 1. Acuerdos de } \\
\text { cooperación entre } \\
\text { entidades de carácter } \\
\text { no estatal: } \\
\text { Organizaciones No } \\
\text { Gubernamentales, } \\
\text { Universidades privadas, } \\
\text { empresas, sindicatos, } \\
\text { centros de investigación } \\
\text { privados, fundaciones, } \\
\text { asociaciones, sector } \\
\text { privado y prestadores } \\
\text { privados de educación } \\
\text { superior, etc. }\end{array}$ \\
\hline $\begin{array}{l}\text { Paradigma } \\
\text { asociado }\end{array}$ & Realismo & $\begin{array}{l}\text { Realismo/ } \\
\text { Gobernanza multinivel }\end{array}$ & Gobernanza multinivel \\
\hline
\end{tabular}

Fuente: Elaboración propia a partir de los trabajos conceptuales consultados

\subsection{La cooperación internacional universitaria}

A partir de la clasificación presentada en el Cuadro $\mathrm{N}^{\circ} 1$, es posible identificar en las categorías de cooperación centralizada indirecta y cooperación descentralizada a las instituciones universitarias. Ahora bien, es en la cooperación descentralizada en la que la universidad aparece como actor autónomo, dando lugar a la cooperación 
Gabriela Michelini Fernando Luján Acosta

Mónica Vivian Ettlin

Modalidades de cooperación internacional universitaria en el contexto de la internacionalización de la educación superior argentina Págs. 86-115

internacional universitaria, definida por las actividades de colaboración y asociación entre instituciones universitarias en temas tan variados como la política y gestión institucional; la formación, la investigación, la extensión y la vinculación para el mutuo fortalecimiento y la proyección institucional; la mejora de la calidad de la docencia; el aumento y la transferencia del conocimiento científico-tecnológico; y la contribución a la cooperación para el desarrollo (Suifi, 2015).

La cooperación internacional universitaria permite la mejora de los modelos "organizativos y estándares de gestión, la formación y especialización de su profesores e investigadores, los contenidos y métodos docentes, la oferta de grado y de posgrado, la participación de profesores de otros países, la movilidad de estudiantes, las actividades de I+D y las actividades de vinculación y extensión" (Sebastián, 2013: 47). De este modo, la cooperación internacional universitaria permite a las instituciones y sus comunidades académicas, fortalecer sus capacidades individuales y proyectarse internacionalmente, incrementando la relación entre cooperación internacional universitaria y la generación de conocimiento (Siufi; 2009: 125).

En este sentido, la tendencia al desarrollo de nuevos modelos de cooperación internacional condiciona y favorece los procesos de internacionalización. De modelos de cooperación espontáneos y con escaso valor añadido y efecto multiplicador, se pasa a modelos que consideran su carácter instrumental y se integran a políticas nacionales o institucionales para la consecución de objetivos estables y sustentables en el conjunto de los sistemas nacionales de innovación (RICYT, 2007: 22).

Además de la cooperación internacional entre universidades, existen proyectos que apuntan a la cooperación universidad-empresa, como por ejemplo, la Red UE-ALCUE entre universidades de América Latina, el Caribe y la Unión Europea (Garrido Noguera et. al., 2013). La cooperación universidad-empresa constituye uno de los lineamientos del Plan Argentina Innovadora 2020.

En este contexto, la cooperación internacional universitaria puede transitar por dos ámbitos: a) la cooperación internacional para el desarrollo y b) la cooperación internacional en educación superior, siendo su ámbito específico la cooperación 
Gabriela Michelini Fernando Luján Acosta

Mónica Vivian Ettlin

Modalidades de cooperación internacional universitaria en el contexto de la internacionalización de la educación superior argentina

Págs. 86-115

internacional universitaria, aspectos que no siempre pueden o suelen diferenciarse con claridad.

Siufi define la cooperación internacional para el desarrollo como "un instrumento que pretende establecer relaciones más justas entre los pueblos y situar a las personas en el centro de todos los esfuerzos, para que cada ser humano pueda desplegar todas sus potencialidades" e implica acciones como "la divulgación, la educación para el desarrollo y la sensibilización" (2009: 125).

En tanto, la cooperación internacional para el desarrollo comprende la movilización de recursos financieros, técnicos y humanos para resolver problemas específicos del crecimiento, fomentar el bienestar y fortalecer las capacidades nacionales (Chávez, 2005: 4-5). La cooperación universitaria al desarrollo supone la colaboración de las universidades en los procesos de desarrollo socioeconómico en dos sentidos: la cooperación bilateral o multilateral exclusivamente entre instituciones universitarias para compartir experiencias y recursos que se trasladen a los procesos de desarrollo en que cada una se encuentre comprometida y la cooperación bilateral o multilateral entre universidades y otros agentes públicos y privados para inducir, fomentar y apoyar estrategias de desarrollo (Chávez, 2005: 6). La cooperación al desarrollo universitario viene consolidándose como una nueva área de actividad en la medida en que se amplían las áreas específicas relacionadas con la docencia, la investigación, la transferencia de conocimiento, que se han establecido previamente como funciones de la universidad. Incluso, se propicia que el conocimiento generado por las universidades sea aplicado o que apunte a una transferencia aplicada o proyección social.

\section{MATERIALES Y MÉTODOS}

Los resultados presentados en este artículo constituyen una caracterización conceptual y tipificación de la cooperación internacional universitaria en Argentina desde sus dimensiones jurídicas e institucionales. Este recorrido fue realizado como parte de un proyecto de investigación más amplio, cuyo objetivo se orientó a caracterizar las operaciones de comunicación de la ciencia involucradas en acciones de cooperación internacional universitaria. 
Gabriela Michelini

Fernando Luján Acosta

Mónica Vivian Ettlin

Modalidades de cooperación internacional universitaria en el contexto de la internacionalización de la educación superior argentina Págs. 86-115

El análisis contempló la revisión de literatura en el campo de la cooperación internacional universitaria y la internacionalización de la educación superior. A partir de las categorías construidas en la revisión bibliográfica, se procedió a la revisión sistemática de documentos a nivel nacional e internacional que abordan la cooperación universitaria internacional.

La elaboración de la muestra en una investigación cualitativa forma parte del análisis, en cuanto es el mismo investigador quien participa en esta selección (Sampieri, 2010). En este caso, la muestra no probabilística de corte teórico fue construida durante la inmersión inicial en el campo, a partir de la selección de documentos que permitían observar indicadores para la caracterización conceptual y tipificación de la cooperación internacional universitaria.

La obtención de los documentos se llevó a cabo a través de la revisión de archivos digitales abiertos en Internet de organismos públicos y la consulta con los organismos correspondientes. Conformaron la muestra:

1. Documentos de organismos nacionales que promueven y supervisan la cooperación universitaria

2. Documentos de organismos internacionales (regionales y transnacionales) que promueven y supervisan la cooperación universitaria.

\section{RESULTADOS}

A partir del análisis de la información relevada fue posible establecer una descripción de la cooperación internacional universitaria:

\subsection{Políticas de la cooperación universitaria}

Las acciones de cooperación universitaria se enmarcan en un entorno legal nacional que las regula. Entre los principales resultados, se destaca que en Argentina, las universidades públicas y privadas suelen adoptar diferentes formas de gestión.

Las universidades públicas son autónomas y creadas por ley, con su propio presupuesto, con capacidad para dictar su propio Estatuto y designar sus propias autoridades. Son organismos descentralizados del Estado, sujetos a las leyes 
Gabriela Michelini

Fernando Luján Acosta

Mónica Vivian Ettlin

Modalidades de cooperación internacional universitaria en el contexto de la internacionalización de la educación superior argentina

Págs. 86-115

nacionales y a las normas de control financiero, pero no a la administración pública. La autonomía universitaria es funcional, en el marco de la gestión de los cometidos educativos y científicos asignados. En el caso argentino, la autonomía universitaria se encuentra plasmada en el texto constitucional (Art. 75 inciso 19) ${ }^{1}$.

Así, en la Argentina, el contenido de la "autonomía y autarquía universitaria" es desarrollado por la Ley de Educación Superior 24.521, señalado su Artículo 29: "Las instituciones universitarias tendrán autonomía académica e institucional" detallando en los incisos a) a ñ) su contenido. A los fines del presente trabajo, se destacan el inciso del Art- $29 \mathrm{~m}$ ) "Mantener relaciones de carácter educativo, científico-cultural con instituciones del país y del extranjero"; y el Artículo 59: "Las instituciones universitarias nacionales tienen autarquía económico-financiera que ejercerán dentro del régimen de la ley 24.156 de Administración Financiera y Sistemas de Control del Sector Público Nacional", precisándose sus facultades en los inciso a) a f), en los que se destaca el inciso e) "Constituir personas jurídicas de derecho público o privado, o participar en ellas".

Las universidades públicas o privadas, nacionales y extranjeras pueden realizar diversos tipos de acuerdo de cooperación que comporten crear o no una nueva persona jurídica, de carácter público o privado. Muchas veces plasman sus modalidades de acción colectiva en estructuras caracterizadas por una amplia plasticidad institucional, funcional y territorial (Diez de Velasco, 2010:45) ${ }^{2}$. Por medio de la firma de acuerdos de voluntades, establecen objetivos y fines, sus derechos y obligaciones de estos ámbitos de cooperación.

Si adoptan una forma más estructurada de cooperación, suelen conformar una asociación civil, asociación simple o fundación en términos del Código Civil y Comercial de la Nación Argentina (CCyCN), aprobado por la Ley 26.994 de 2015,

\footnotetext{
${ }^{1}$ Constitución de la Nación Argentina (1994): Art 75 inciso 19 (parte específica) “(...) Sancionar leyes de organización y de base de la educación que consoliden la unidad nacional respetando las particularidades provinciales y locales; que aseguren la responsabilidad indelegable del Estado, la participación de la familia y la sociedad, la promoción de los valores democráticos y la igualdad de oportunidades y posibilidades sin discriminación alguna; y que garanticen los principios de gratuidad y equidad de la educación pública estatal y la autonomía y autarquía de las universidades nacionales."

${ }^{2} \mathrm{Si}$ bien Diez de Velasco utiliza esta caracterización para referirse a diferentes organizaciones internacionales no gubernamentales, la descripción le cabe perfectamente al modo en que operan y se desenvuelve la cooperación universitaria internacional.
} 
Gabriela Michelini Fernando Luján Acosta

Mónica Vivian Ettlin

Modalidades de cooperación internacional universitaria en el contexto de la internacionalización de la educación superior argentina Págs. 86-115

constituyendo una persona (Título II. Capítulo 2, art. 168 y ss. CCyCN). En el Capítulo 16 del $\mathrm{CCyCN}$, que regula diferentes "contratos asociativos", entre los cuales se presentan negocios en participación, agrupaciones de colaboración, uniones transitorias, consorcios de cooperación; lo que posibilita, en estos supuestos de "comunidad de fin", encontrar una forma jurídica de actuación, sin crear una sociedad al respecto (Art. 1442). A estos contratos no se les aplican las normas sobre sociedades, no se constituyen personas jurídicas, sociedades ni sujetos de derecho; tienen libertad de forma (Art. 1444), libertad de contenidos (Art. 1446) y pueden estar o no inscriptos (Art. 1447). En este contexto, se asumen diferentes modelos de cooperación internacional entre universidades; y entre universidades y otras organizaciones, también comienzan a perfilarse modos de cooperación universidadempresa.

En tanto, la Subsecretaría de Gestión y Coordinación de Políticas Universitarias del Ministerio de Educación de la Nación lleva adelante el "Programa de Internacionalización de la Educación Superior y Cooperación Internacional" con el objetivo general de maximizar el aprovechamiento de las oportunidades en el mundo de la cooperación educativa y académica, en el ámbito nacional, regional e internacional.

En el ámbito argentino, ello se canaliza a través del Consejo Interuniversitario Nacional (CIN) en particular a través de la Red de Cooperación internacional de Universidades Nacionales (REDCIUN)-. Las acciones de cooperación bilateral tienen como eje estratégico la cooperación con los países de América Latina y del MERCOSUR en particular. En cuanto a la cooperación multilateral el eje estratégico se encuentra identificado en el MERCOSUR y la UNASUR.

El Programa representa a la Argentina en las reuniones de la Comisión Regional Coordinadora de Educación Superior del Sector Educativo del MERCOSUR y en las reuniones del Consejo Suramericano de Educación, Cultura, Ciencia, Tecnología e Innovación de UNASUR (COSECCTI) a través del Subgrupo de Educación Superior. También toma participación el Espacio Iberoamericano del Conocimiento.

Podemos citar, en este sentido, dos acciones específicas que son consecuencia de la 
Gabriela Michelini Fernando Luján Acosta

Mónica Vivian Ettlin

Modalidades de cooperación internacional universitaria en el contexto de la internacionalización de la educación superior argentina Págs. 86-115

aplicación de estas políticas en Argentina: la creación de RIACES (Red Iberoamericana de Acreditación de Calidad de la Educación Superior) y el sistema ARCU- SUR, en el marco del MERCOSUR- Educativo.

RIACES fue fundado por iniciativa de ANECA y CONEAU, entre otras agencias, inspirados por la pertenencia a INQAAHE. La asamblea fundacional se realizó en Buenos Aires en 2003, integrada por ministerios o secretarías de educación superior, comisiones nacionales y agencias nacionales de los países miembro, que solo cuentan con un voto. La agencia promueve encuentros y movilidad para la formación técnica, cooperación horizontal, con apoyo económico de la UNESCO y el Banco Mundial.

Por su parte, el Sistema de Acreditación Regional de Carreras Universitarias es resultado de un Acuerdo entre los Ministros de Educación de Argentina, Brasil, Paraguay, Uruguay, Bolivia y Chile, homologado por el Consejo del Mercado Común del MERCOSUR a través de la Decisión CMC $n^{\circ}$ 17/08. Ejecuta la evaluación y acreditación de carreras universitarias y se gestiona a través de la Red de Agencias Nacionales de Acreditación en el ámbito del Sector Educativo del MERCOSUR. Para ello, respeta las legislaciones de cada país y la autonomía de las instituciones universitarias y evalúa solo carreras de grado que cuenten con reconocimiento oficial en su país y que tengan egresados. Su objetivo final es ofrecer la garantía pública, entre los países de la región, del nivel académico y científico de las carreras. El nivel académico se define según criterios y perfiles tanto o más exigentes que los aplicados por los países en sus instancias nacionales análogas.

Respecto de las acciones que encaran las propias universidades, como entidades autónomas y autárquicas en un marco creciente de oportunidades e instrumentos para la cooperación internacional, se puede señalar que cada institución construye sus propias políticas de vinculación institucional, nacionales e internacionales. Muchas veces basadas en la mayor afinidad o facilidad de acceso a sus recursos. Es por ello que cabría analizar o verificar si existe una planificación clara y definida en materia de cooperación internacional, un seguimiento de los beneficios de las opciones efectuadas, y en qué medida se conocen o se están aprovechando las mejores opciones disponibles (Knight, 2011). 
Gabriela Michelini Fernando Luján Acosta Mónica Vivian Ettlin Modalidades de cooperación internacional universitaria en el contexto de la internacionalización de la educación superior argentina Págs. 86-115

En igual sentido Sebastián señala que si la cooperación responde a un modelo estratégico es posible la búsqueda de las mejores oportunidades para la cooperación, "en especial en cuanto al aprovechamiento de los programas existentes de financiación de la cooperación, los objetivos perseguidos y las contrapartes deseables o posibles" (Sebastián, 2013: 47). En cambio, cuando la cooperación nace de forma espontánea por los vínculos preexistentes o por las oportunidades de financiamiento, entonces se establecen relaciones multipolares que pueden ir en beneficio o detrimento de la misma institución.

\subsection{Modalidades de la cooperación universitaria internacional}

Como entidades autónomas, las universidades pueden asumir distintas formas bajo las cuales se formaliza la cooperación internacional. Estas modalidades también pueden encontrarse en los programas e instrumentos de la cooperación centralizada.

- Convenios de asistencia técnica y/o capacitación, y convenios de cooperación académica

Las universidades suelen contener normativas internas que establecen las disposiciones en base a las cuales desarrollar actividades de asistencia técnica y/o capacitación, cooperación académica y acuerdos tecnológicos, tanto con entidades públicas o privadas, nacionales o extranjeras. Entonces, debe verificarse en la normativa correspondiente a cada universidad, los alcances y posibilidad de la cooperación universitaria.

Por su parte, los convenios de cooperación técnica internacional pueden estar en el marco de un acuerdo con un organismo internacional, cuyo tratado deberá ser aprobado por ley, como el Programa de las Naciones Unidas para el Desarrollo (PNUD), aprobado por la Ley $N^{\circ} 23.396$.

- Convenios de cooperación científica- tecnológica y los convenios de investigación y desarrollo $(I+D)$

Por fuera de las modalidades señaladas también es posible la suscripción de diferentes tipos de acuerdos de cooperación técnica, asesoramiento y asistencia técnica, que apuntan a fortalecer la investigación científica en la Universidad, procurando una investigación orientada a la producción de conocimiento tecnológico, 
Gabriela Michelini Fernando Luján Acosta

Mónica Vivian Ettlin

Modalidades de cooperación internacional universitaria en el contexto de la internacionalización de la educación superior argentina

Págs. 86-115

que fomenta vínculos con sectores que demandan tal conocimiento científico, a la vez que mejoran los ingresos del personal docente investigador.

Esta transferencia aplicada, se realiza a requerimiento de entidades externas. De este modo el conocimiento generado en las universidades llegue al entorno socioeconómico. Tal el caso de la Resolución 1655/1985 del Consejo Superior de la Universidad de Buenos Aires, que aprueba la reglamentación para trabajos de alta especialización técnica, tareas de transferencias de conocimientos tecnológicos y prestación de servicios a terceros, con financiamiento total o parcial de éstos. Estas actividades $\mathrm{I}+\mathrm{D}$, pueden adoptar la modalidad de "servicios a terceros" (realización de mediciones, análisis, evaluaciones, etc.); "convenios específicos" (estudios, investigaciones, desarrollos, tareas de transferencia de conocimientos tecnológicos con un objeto concreto) y "convenios generales" (que permiten la suscripción de convenios específicos). Se especifica en la normativa los porcentajes de participación entre los docentes investigadores involucrados en dicho acuerdo; también puede ser una investigación colaborativa realizada entre la universidad y la empresa, con porcentajes a repartir entre ambas.

En otras convocatorias públicas se establece la posibilidad de que empresas y universidades sean socias en la realización de un proyecto conjunto, en el que ambas aportan conocimientos y recursos propios, y comparten objetivos, riesgos, beneficios y la titularidad de los resultados.

En el ámbito internacional se orienta además a otros tipos de transferencia de conocimiento en las universidades como las licencias de tecnología y know how; la creación de empresas de base tecnológica; las patentes u otros tipos de protección del conocimiento generado en las universidades mediante la investigación, etc.

- Conformación de organizaciones, instituciones y asociaciones internacionales. Configuración de redes.

La cooperación universitaria internacional ha constituido, más allá de los convenios bilaterales de cooperación señalados, un entramado institucional evidenciado en las diferentes formas de vinculación entre organizaciones, instituciones y asociaciones, internacionales o multinacionales, que relaciona a las universidades en forma 
Gabriela Michelini

Fernando Luján Acosta

Mónica Vivian Ettlin

Modalidades de cooperación internacional universitaria en el contexto de la internacionalización de la educación superior argentina Págs. 86-115

cooperativa, para el logro de objetivos comunes, persiguiendo variados propósitos y con un amplio abanico de áreas y ámbitos de actuación.

Estas formas de cooperación universitaria han sido definidas por el Instituto Internacional de la Unesco para la Educación Superior en América Latina y el Caribe (IESALC-UNESCO) como "redes universitarias", definidas como "organizaciones, instituciones o asociaciones, internacionales o multinacionales que conforman un conjunto integrado por diversas instituciones relacionadas entre sí de manera horizontal y autónoma (no de dependencia ni piramidal) que persiguen propósitos específicos y comunes; (...) un entramado de mecanismos de comunicación entre instituciones interrelacionadas de manera permanente y multidireccional" (Ramos, 2007:4). Se reserva la noción de "red" para vinculaciones no institucionalizadas de la cooperación universitaria. La clasificación en base a la cual trabaja el IESALC/UNESCO ${ }^{3}$, las agrupa según su alcance y temática: Redes regionales; Redes nacionales; Redes temáticas y Agencias y organismos internacionales.

En este sentido, Huerta (2008) propone otra clasificación para abordar las redes universitarias en América Latina:

- Redes generalistas: son aquellas que tienen como objeto generar instancias e instrumentos de expansión, intercambio o participación. Incluye como ejemplos de este tipo de redes la Asociación Internacional de Universidades (AIU); la Unión de Universidades de América Latina (UDUAL); y otras con programas más generales, como el European Consortium of Innovative Universties (ECIU) o la Red de Universidades de las Capitales de Europa (UNICA).

- Redes específicas: son aquellas cuyos miembros se reúnen con un objetivo operativo. Cita como ejemplo el Programa ERASMUS y el programa ALFA (América Latina- Formación Académica). Dentro de esta categoría, menciona a aquellas Redes específicas dedicadas a América Latina en Europa, como pueden ser la Associazione di Studi Latinoamericani (ASSLA) Italia, o la Society for Latin American Studies (SLAS) del Reino Unido, entre otras. También analiza las "asociaciones latino- americanistas de investigación con vocación regional", que también incluyen a investigadores

\footnotetext{
3 La información se encuentra disponible en: http://www.iesalc.unesco.org.ve/index.php?option=com content\&view=article\&id=2317\&ltemid=795\&lang =es
} 
Gabriela Michelini

Fernando Luján Acosta

Mónica Vivian Ettlin

Modalidades de cooperación internacional universitaria en el contexto de la internacionalización de la educación superior argentina

Págs. 86-115

europeos, como la Red Europea de información y de documentación sobre América Latina (REDIAL).

Las clasificaciones remiten a acciones institucionalizadas. Sin embargo, existen en el ámbito académico también bajo la denominación de red las vinculaciones sin formalización, como es el caso de los llamados Colegios Invisibles. Es por ello que se puede afirmar que también existen acciones no reguladas que integran acciones e interacciones de actores, grupos de individuos, basados más en lo espontáneo y dinámico de las interacciones, que pueden aparecer en un momento dado, y en un cierto contexto definido por la presencia de ciertas prácticas más o menos formalizadas. En algunos casos, estos vínculos informales pueden generar asociaciones institucionalizadas.

No obstante, los vínculos de la cooperación internacional universitaria, integran siempre una instancia de formalización que las valida. De otra forma, no resulta posible realizar la medición o el seguimiento de esas instancias de cooperación por fuera de las metodologías indirectas, como la medición de los artículos publicados en coautoría.

A veces, las modalidades de cooperación de acuerdo a quienes las conforman son redes de información y comunicación, apoyadas en la tecnología por las cuales sus integrantes intercambian información, conocimientos, resultados científicos con mayor o casi ninguna institucionalización; o pueden ser "redes académicas" centradas en la movilidad e intercambio de de estudiantes y docentes, estudios de posgrado, etc. Pueden estar conformadas por las universidades, alguno de sus departamentos, o por docentes e investigadores universitarios. También existen las "redes temáticas", las "redes de investigación", las "redes de innovación".

En cuanto al ámbito geográfico pueden ser regionales, globales, siendo formales o informales de acuerdo a su institucionalización o no.

Lo cierto es que estas formas de cooperación "están generando un nuevo modo de multilateralidad basada en la funcionalidad y la flexibilidad", asimismo frente "a la visión tradicional de la multilateralidad en el ámbito de la cooperación internacional, cristalizada fundamentalmente a través de Organismos Intergubernamentales, con el 
Gabriela Michelini

Fernando Luján Acosta

Mónica Vivian Ettlin

Modalidades de cooperación internacional universitaria en el contexto de la internacionalización de la educación superior argentina

Págs. 86-115

peso de sus burocracias y la complejidad en la toma de decisiones, las redes de cooperación suponen una alternativa que se caracteriza por la simplicidad organizativa y la diversificación en los objetivos y procedimientos". (Sebastián, 2004: 10).

\subsection{La Cooperación universitaria en Iberoamérica}

La cooperación universitaria institucionalizada en Iberoamérica se desarrolla en el marco de la Organización de Estados Iberoamericanos para la Educación, la Ciencia y la Cultura (OEI), de acuerdo a lo definido por Sebastián como "cooperación universitaria iberoamericana en sentido estricto" (2013: 49). En el Cuadro № 2 se describen distintos entornos de cooperación.

\section{Cuadro $N^{\circ}$ 2. Entornos de cooperación en el ámbito iberoamericano.}

\begin{tabular}{|c|c|}
\hline Entorno & Descripción \\
\hline $\begin{array}{l}\text { Programa de Intercambio y } \\
\text { Movilidad Académica de la } \\
\text { Organización de los Estados } \\
\text { lberoamericanos (PIMA) } \\
\text { Fuente: } \\
\text { http://www.oei.es/pima/ }\end{array}$ & $\begin{array}{l}\text { Iniciativa de movilidad de estudiantes de grado. Está estructurado } \\
\text { en redes temáticas conformadas por instituciones de educación } \\
\text { superior de al menos tres países participantes en el programa, } \\
\text { con garantía de reconocimiento, por parte de la universidad de } \\
\text { origen, de los estudios realizados por los estudiantes en otra } \\
\text { universidad de la red. }\end{array}$ \\
\hline $\begin{array}{l}\text { Espacio Iberoamericano del } \\
\text { Conocimiento } \\
\text { Fuente: } \\
\text { http://www.oei.es/historico/espaci } \\
\text { oiberoamericanodelconocimiento. } \\
\text { htm }\end{array}$ & $\begin{array}{l}\text { Creado por la XV Cumbre Iberoamericana de Jefes de Estado y } \\
\text { Gobierno (2005) con el propósito de incrementar los niveles de } \\
\text { desarrollo de la región, disminuir la brecha entre los países, } \\
\text { garantizando un incremento de la productividad, brindando mejor } \\
\text { calidad y accesibilidad a los bienes y servicios por parte de la } \\
\text { población e incrementando la competitividad internacional de la } \\
\text { región. En lo relativo a la educación superior se plantea la } \\
\text { creación de un espacio universitario común, integrado, de } \\
\text { cooperación universitaria y de convergencia. Los criterios que lo } \\
\text { guían son la cooperación solidaria para la atención de las } \\
\text { asimetrías, el respeto de la diversidad, la mejora continua y el } \\
\text { aseguramiento de la calidad y pertinencia de la educación } \\
\text { superior, la investigación y la innovación como fundamento para } \\
\text { el desarrollo sostenible y la conservación de la biodiversidad en la } \\
\text { región, la complementariedad y la concertación entre los diversos } \\
\text { actores involucrados. En su Hoja de Ruta se establecen tres } \\
\text { grandes líneas de acción: } \\
\text { - El fortalecimiento de los sistemas nacionales y regionales de } \\
\text { acreditación y evaluación de la calidad de la educación superior } \\
\text { - La movilidad académica de estudiantes, profesores e } \\
\text { investigadores } \\
\text { - El desarrollo de acciones en materia de investigación, desarrollo } \\
\text { y transferencia del conocimiento. }\end{array}$ \\
\hline $\begin{array}{l}\text { Programa Pablo Neruda. } \\
\text { Fuente: } \\
\text { http://www.espaciodelcono- } \\
\text { cimiento.org/neruda/ }\end{array}$ & $\begin{array}{l}\text { Es un programa de movilidad académica de postgrado, de ámbito } \\
\text { regional y de carácter multilateral, creado por la XVII Cumbre } \\
\text { Iberoamericana de Jefes de Estado y de Gobierno en Chile (2007) } \\
\text { dentro del Espacio Iberoamericano del Conocimiento (EIC). Está } \\
\text { estructurado en redes temáticas conformadas por instituciones de } \\
\text { educación superior de al menos tres países iberoamericanos } \\
\text { participantes. Cada país define las áreas científicas prioritarias y }\end{array}$ \\
\hline
\end{tabular}


Gabriela Michelini

Fernando Luján Acosta

Mónica Vivian Ettlin

Modalidades de cooperación internacional universitaria en el contexto de la internacionalización de la educación superior argentina

Págs. 86-115

\begin{tabular}{|c|c|}
\hline & $\begin{array}{l}\text { hace público el listado de programas de postgrado que podrán } \\
\text { participar. La condición para la participación de dichos programas } \\
\text { es que cuenten con la acreditación de la calidad de sus } \\
\text { respectivas agencias nacionales }\end{array}$ \\
\hline $\begin{array}{l}\text { Red Iberoamericana para la } \\
\text { Acreditación de la Calidad de la } \\
\text { Educación Superior (RIACES) } \\
\text { Fuente: } \underline{\text { http://riaces.org/ }}\end{array}$ & $\begin{array}{l}\text { Se constituye como instancia para promover entre los países } \\
\text { iberoamericanos la cooperación y el intercambio en materia de } \\
\text { evaluación y acreditación de la calidad de la educación superior, y } \\
\text { contribuir así a la garantía de la calidad de la educación superior } \\
\text { de estos países. }\end{array}$ \\
\hline $\begin{array}{l}\text { Consejo } \\
\text { lberoamericano (CUIB) } \\
\text { Fuente: } \\
\text { http://www.cuib.org/protocolo con } \\
\text { stitucion cuib.pdf }\end{array}$ & $\begin{array}{l}\text { Organización no gubernamental que se configura como una red } \\
\text { de redes de las universidades iberoamericanas creado en } 2002 \\
\text { en Cartagena de Indias. Está integrado por las organizaciones } \\
\text { nacionales representativas de las universidades y otras } \\
\text { instituciones de educación superior de: Argentina, Bolivia, Brasil, } \\
\text { Chile, Colombia, Costa Rica, Cuba, Ecuador, El Salvador, } \\
\text { España, Guatemala, Honduras, México, Nicaragua, Panamá, } \\
\text { Paraguay, Perú, Portugal, República Dominicana, Uruguay y } \\
\text { Venezuela. }\end{array}$ \\
\hline $\begin{array}{l}\text { Observatorio Iberoamericano de } \\
\text { la Ciencia, la Tecnología y la } \\
\text { Sociedad (Observatorio CTS) } \\
\text { Fuente: } \\
\text { http://www.observatoriocts.org/ }\end{array}$ & $\begin{array}{l}\text { Fue creado en } 2008 \text { con la misión de desarrollar un programa de } \\
\text { estudios estratégicos en Ciencia, Tecnología y Sociedad que } \\
\text { indague en las fronteras de la ciencia y de las demandas sociales } \\
\text { de los pueblos de lberoamérica. Está asociado desde } 2009 \text { con la } \\
\text { Red de Indicadores de Ciencia y Tecnología (RICYT). Entre } 2009 \\
\text { y 2012, el Observatorio CTS contó además con el patrocinio de la } \\
\text { Agencia Española de Cooperación Internacional para el } \\
\text { Desarrollo (AECID). }\end{array}$ \\
\hline $\begin{array}{l}\text { Programa CYTED } \\
\text { Fuente: http://www.cyted.org/ }\end{array}$ & $\begin{array}{l}\text { Es una plataforma que promueve y da soporte a la cooperación } \\
\text { multilateral en ciencia y tecnología, orientada a la transferencia de } \\
\text { conocimientos, experiencias, información, resultados y } \\
\text { tecnologías entre los países de la región Iberoamericana. Fue } \\
\text { creado en } 1984 \text { mediante un acuerdo marco interinstitucional } \\
\text { firmado por Argentina, Bolivia, Brasil, Chile, Colombia, Costa } \\
\text { Rica, Cuba, Ecuador, El Salvador, España, Guatemala, Honduras, } \\
\text { México, Nicaragua, Panamá, Paraguay, Perú, Portugal, } \\
\text { República Dominicana, Uruguay y Venezuela. Desde 1995, el } \\
\text { Programa CYTED se encuentra formalmente incluido entre los } \\
\text { Programas de Cooperación de las Cumbres Iberoamericanas de } \\
\text { Jefes de Estado y de Gobierno. Son objetivos específicos del } \\
\text { Programa CYTED: } \\
\text { - Fomentar la integración de la Comunidad Científica y } \\
\text { Tecnológica Iberoamericana, promoviendo una agenda de } \\
\text { prioridades compartidas para la Región. } \\
\text { - Fortalecer la capacidad de desarrollo tecnológico de } \\
\text { Iberoamérica mediante la promoción de la investigación científica } \\
\text { conjunta, la transferencia de conocimientos y técnicas, y el } \\
\text { intercambio de científicos y tecnólogos entre grupos de I+D+l de } \\
\text { los países miembros. } \\
\text { - Promover la participación de sectores empresariales de los } \\
\text { países miembros interesados en los procesos de innovación, en } \\
\text { concordancia con las investigaciones y desarrollos tecnológicos } \\
\text { de la Comunidad Científica y Tecnológica lberoamericana. } \\
\text { - Promover la participación de los investigadores de la Región en } \\
\text { otros programas multilaterales de investigación a través de } \\
\text { acuerdos. }\end{array}$ \\
\hline $\begin{array}{l}\text { Red de Indicadores de Ciencia y } \\
\text { Tecnología (RICYT) } \\
\text { Fuente: http://www.ricyt.org/ }\end{array}$ & $\begin{array}{l}\text { La Red de Indicadores de Ciencia y Tecnología Iberoamericana e } \\
\text { Interamericana (RICYT), de la que participan todos los países de } \\
\text { América, junto con España y Portugal, fue adoptada por el } \\
\text { CYTED como red iberoamericana y por la Organización de los } \\
\text { Estados Americanos (OEA) como red interamericana. Su puesta } \\
\text { en marcha se hizo efectiva a fines de abril de 1995. El objetivo }\end{array}$ \\
\hline
\end{tabular}


Gabriela Michelini

Fernando Luján Acosta

Mónica Vivian Ettlin

Modalidades de cooperación internacional universitaria en el contexto de la internacionalización de la educación superior argentina

Págs. 86-115

\begin{tabular}{|l|l|}
\hline & $\begin{array}{l}\text { general de la RICYT es promover el desarrollo y el uso de } \\
\text { instrumentos para la medición y el análisis de la ciencia y la } \\
\text { tecnología en Iberoamérica, en un marco de cooperación } \\
\text { internacional, con el propósito de profundizar en su conocimiento } \\
\text { y su utilización como instrumento político para la toma de } \\
\text { decisiones. }\end{array}$ \\
\hline $\begin{array}{l}\text { Asociación } \\
\text { lberoamericana de Postgrado } \\
\text { (AUIP) }\end{array}$ & $\begin{array}{l}\text { Es un organismo internacional no gubernamental sin fines de } \\
\text { Fuente: } \\
\text { lucro, dedicado a la cooperación académica interuniversitaria, en } \\
\text { el marco de la Comunidad lberoamericana de Naciones. } \\
\text { Tiene como fin el fomento y el desarrollo de los estudios de } \\
\text { postgrado y doctorado, contribuyendo a la formación de } \\
\text { profesores universitarios, científicos y profesionales, en función de } \\
\text { las necesidades de desarrollo de cada país y de la comunidad } \\
\text { iberoamericana de naciones. Cuenta con 197 entidades } \\
\text { asociadas. }\end{array}$ \\
\hline
\end{tabular}

Fuente: elaboración propia en base a datos secundarios.

La descripción de entornos de cooperación que ofrece el Cuadro $\mathrm{N}^{\circ} 2$, aunque no exhaustiva, permite observar que existe una elevada institucionalización de las acciones. Por ejemplo, si se agrupan estos entornos de cooperación de acuerdo a las modalidades definidas, se observa que el Estado asume un rol protagónico en la dinámica de esos ámbitos.

\section{Cuadro №3. Entornos de cooperación en el ámbito iberoamericano de acuerdo a las modalidades}

\begin{tabular}{|c|c|c|c|}
\hline \multirow[t]{2}{*}{ Modalidades } & \multicolumn{2}{|c|}{ Cooperación estatal } & \multirow{2}{*}{$\begin{array}{l}\text { Cooperación no } \\
\text { estatal } \\
\text { Descentralizada }\end{array}$} \\
\hline & Centralizada & $\begin{array}{l}\text { Centralizada } \\
\text { indirecta }\end{array}$ & \\
\hline Ámbitos y organizaciones & $\begin{array}{l}\text { - Programa } \\
\text { CYTED } \\
\text { - Espacio } \\
\text { Iberoamericano } \\
\text { del Conocimiento } \\
\text { (EIC) }\end{array}$ & $\begin{array}{l}\text { Consejo } \\
\text { Universitario } \\
\text { Iberoamericano } \\
\text { (CUIB) }\end{array}$ & $\begin{array}{l}\text { Asociación } \\
\text { Universitaria } \\
\text { lberoamericana } \\
\text { de Postgrado } \\
\text { (AUIP) }\end{array}$ \\
\hline Cooperación académica & $\begin{array}{l}\text { - Programa de } \\
\text { Intercambio y } \\
\text { Movilidad } \\
\text { Académica de la } \\
\text { Organización de } \\
\text { los Estados } \\
\text { Iberoamericanos } \\
\text { (PIMA) } \\
\text { - Observatorio } \\
\text { lberoamericano } \\
\text { de la Ciencia, la } \\
\text { Tecnología y la } \\
\text { Sociedad } \\
\text { (Observatorio } \\
\text { CTS) }\end{array}$ & & \\
\hline
\end{tabular}


Gabriela Michelini

Fernando Luján Acosta

Mónica Vivian Ettlin

Modalidades de cooperación internacional universitaria en el contexto de la internacionalización de la educación superior argentina

Págs. 86-115

\begin{tabular}{|c|c|}
\hline & $\begin{array}{l}\text { - Programa Pablo } \\
\text { Neruda }\end{array}$ \\
\hline Redes & $\begin{array}{l}\text { - Red de } \\
\text { Indicadores de } \\
\text { Ciencia y } \\
\text { Tecnología } \\
\text { (RICYT) } \\
\text { - Red } \\
\text { lberoamericana } \\
\text { para la } \\
\text { Acreditación de la } \\
\text { Calidad de la } \\
\text { Educación } \\
\text { Superior } \\
\text { (RIACES) }\end{array}$ \\
\hline
\end{tabular}

Fuente: elaboración propia sobre la base de datos secundarios.

En el cuadro $N^{\circ} 3$, se observa una mayor cantidad de ámbitos de cooperación con presencia directa del Estado que involucran a instituciones del ámbito universitario. Este factor puede asociarse a que, por un lado, la existencia de redes y otras formas descentralizadas de cooperación son sistematizables a partir de que asumen cierta formalidad, de modo tal que impacta en la clasificación según modalidades que ofrece el cuadro $\mathrm{N}^{0} 3$. Pero también es posible asociar esta disparidad con la relevancia que la política pública asume para promover, garantizar y dar sostenibilidad en el tiempo a la cooperación internacional universitaria. Asimismo, otro aspecto que se visualiza en este análisis es que las modalidades incluyen instrumentos heterogéneos, de modo tal que su definición es laxa y otorgan flexibilidad en la construcción de herramientas viables para el desarrollo de la cooperación.

\section{CONCLUSIONES}

El presente artículo realiza una descripción de la cooperación internacional universitaria como una acción de la internacionalización de la educación superior. La internacionalización en el contexto contemporáneo es un proceso que integra dimensiones internacionales, interculturales y globales en el objetivo, función y provisión de la educación superior.

Desde el enfoque de la internacionalización de la educación superior, las 
Gabriela Michelini Fernando Luján Acosta

Mónica Vivian Ettlin

Modalidades de cooperación internacional universitaria en el contexto de la internacionalización de la educación superior argentina Págs. 86-115

universidades se presentan como actores del mundo global, con impacto local y nacional. Esta presencia de la universidad en el entorno internacional se ve favorecida por la consolidación de la economía del conocimiento en un contexto de globalización, en el que los estados perdieron parte de su relevancia en el ámbito internacional a medida que otros actores, fundamentalmente las empresas y los mercados, se convirtieron en actores clave de los intercambios a nivel global. No obstante, el artículo se ha centrado en la cooperación internacional universitaria, que en coincidencia con la literatura sobre el tema se ha asociado a los modelos solidarios de internacionalización, frente a los modelos de competencia que se basan en prácticas de mercado para la internacionalización de la educación superior.

Una vez que se identificó a la cooperación internacional universitaria como una acción específica de la internacionalización de la educación superior, el trabajo diferenció modalidades de cooperación internacional universitaria, entre las cuales se distinguen: a) Convenios de asistencia técnica y/o capacitación y convenios de cooperación académica; b) Convenios de cooperación científica-tecnológica y los convenios de investigación y desarrollo (I+D); c) Conformación de organizaciones, instituciones y asociaciones internacionales y configuración de redes, que pueden ser de carácter gubernamental o no gubernamental.

Las modalidades de cooperación dan cuenta de las acciones que emprenden las instituciones universitarias como parte de su propia internacionalización. Se puede observar que existe una correspondencia entre estas tres modalidades de cooperación y las funciones propias de la universidad: la función de formación y docencia se corresponde con las acciones de la modalidad a) de asistencia técnica y cuestiones académicas; la función de investigación se ve articulada con la modalidad b) de cooperación, referida a las actividades de producción de conocimiento. Finalmente, la función de extensión o de vinculación social de la universidad, se corresponde con la modalidad c) de cooperación, referida a la instauración de entidades internacionales que potencian los resultados de las funciones primordiales de la universidad.

Luego, se definieron entornos de colaboración a nivel iberoamericano, en el marco de los cuales pueden darse distintas modalidades de cooperación internacional universitaria. Estos entornos pueden ser abordados según los criterios de cooperación 
Gabriela Michelini

Fernando Luján Acosta

Mónica Vivian Ettlin

Modalidades de cooperación internacional universitaria en el contexto de la internacionalización de la educación superior argentina Págs. 86-115

internacional basados en el nivel del Estado: centralizado directo, centralizado indirecto y descentralizado (realizado por los agentes no estatales). Este criterio de análisis es pertinente para abordar la internacionalización de la educación superior desde el enfoque de los estados nacionales, predominante en la literatura latinoamericana sobre el tema.

A partir del análisis de las modalidades predominantes de cooperación en el entorno iberoamericano se observó que en el mismo predominan las acciones promovidas por los Estados antes que por las universidades de forma autónoma, aunque la Ley argentina prevé la autonomía de las instituciones universitarias en la dimensión académica. Por lo tanto, cabe suponer que en el entorno iberoamericano, los Estados retienen un rol relevante en la promoción de la cooperación universitaria y en este sentido ofrecen ámbitos posibles de acción para las instituciones universitarias y sus miembros. Pero al mismo tiempo, cabe preguntarse si esta predominancia no se vería transformada al analizar modalidades no formalizadas en el ámbito iberoamericano, y qué impacto tendría también la inclusión de acciones de internacionalización relativas a las prácticas de mercado.

\section{BIBLIOGRAFÍA}

AEBISCHER, P. (2015) "Universities: increasingly global players", en UNESCO Science Report, UNESCO, París, Francia.

ALTBACH, P. G., REISBERG, L., \& RUMBLEY, L. E. (2009) Trends in Global Higher Education: Tracking an Academic Revolution, UNESCO, París, Francia.

ARGENTINA (1994) Constitución de la Nación argentina. Ediciones del país, Longchamps, Argentina.

ARGENTINA (2002). Congreso de la Nación, Ley de educación superior, modificación de la ley 24.521, ley 25.573, 11 de abril de 2002, en: http://servicios.infoleg.gob.ar/infoleglnternet/anexos/70000-74999/73892/norma.htm

(2014). Congreso de la Nación, Código Civil y Comercial de la Nación, ley 26.994, 1 de octubre de 2014, en: http://servicios.infoleg.gob.ar/infoleglnternet/verNorma.do?id=235975

(2015). Congreso de la Nación, Ley de implementación efectiva de la responsabilidad del estado en el nivel de educación superior, modificatoria ley 24.521, ley 27.204, 28 de octubre de 2015, en: 
http://servicios. infoleg.gob.ar/infolegInternet/verNorma.do?id=254825

AZEVEDO, M.L.N. (2015) "Internacionalização ou transnacionalização da educação superior: entre a formação de um campo social global e um mercado de ensino mundializado", Crítica Educativa, Vol.1, n.1, p. 56-79. Disponible: http://www.criticaeducativa.ufscar.br/index.php/criticaeducativa/article/view/24 (Consulta realizada 09/06/2017).

BARSKY, O.; CORENGIA, A.; FLIGUER, J.; MICHELINI, G. (2016) La investigación en la universidad privada argentina, CRUP, Buenos Aires, Argentina.

BENEITONE, P. (2014): "De la Cooperación Internacional Universitaria a la Internacionalización de la Educación Superior: ¿cambio de paradigma o maquillaje conceptual?" en: Tangelson, G. (Comp.). Desde el sur: miradas sobre la internacionalización. (pp. 29-38) Remedios de Escalada: Ediciones de la UNLa Universidad Nacional de Lanús, Argentina.

CONEAU (2012) La CONEAU y el sistema universitario argentino. Autor, Buenos Aires, Argentina, disponible: http://www.coneau.gov.ar/archivos/publicaciones/documentos/La CONEA $\underline{\mathrm{U}}$ y el sistema universitario argentino.pdf (consulta realizada 21/11/2016)

CORENGIA, A. (2015) El impacto de la CONEAU en universidades argentinas: estudio de casos. Teseo, Buenos Aires, Argentina.

DÁVILA, M. (2008) "Tendencias internacionales de la educación superior", Revista Sudamericana de Educación, Universidad y Sociedad, Volumen 1, № 1-2, disponible: http://www.ub.edu.ar/investigaciones/dt nuevos/219 davila.pdf (consulta realizada 21/10/2016)

DE WIT, H. (2010) Internationalisation of Higher Education in Europe and its assessment, trends and issues. The Hague: NVAO, disponible: https://nvao.com/system/files/pdf/Internationalisation\%20of\%20Higher\%20Education\% 20in\%20Europe\%20Hans\%20de\%20Wit\%202010.pdf (consulta realizada 21/11/2916)

DIDOU AUPETIT, S. (2009) Fuga de cerebros, movilidad académica y redes científicas. Perspectivas latinoamericanas, Cinvestav, México D.F., México.

(2007) "La internacionalización de la educación superior en América Latina: oportunidades y desafíos". Conferencia dictada en la Universidad Nacional de Córdoba, Argentina. ABELEDO, C.R. (2004) "La investigación en la universidad: ¿Creación de conocimientos o desarrollo tecnológico?”, en: Pugliese, J. C. (Ed.) Universidad, Sociedad y Producción, Ministerio de Educación, Ciencia y Tecnología, Secretaría de Políticas Universitarias, Buenos Aires, Argentina.

ENDERS, J. (2004) "Higher education, internationalisation, and the nation-state: Recent developments and challenges to governance theory". Higher Education. Volumen 47, Número 3, pp. 361-382.

GACEL-ÁVILA, J. (2005) "La internacionalización de la Educación Superior en 
América Latina: el caso de México" Cuaderno de Investigación en la Educación. No 20 , disponible: http://www.uco.edu.co/ova/OVA\%20Internacionalizacion/ova\%20internacionalizaci\%C 3\%B3n/g)\%20Gacel-\%C3\%81vila, \%20J\%20Cuaderno $\% 20$ de $\% 20$ Investigaci $\%$ C3\%B3n $\% 20$ en $\% 20$ la $\% 20$ Educaci\%C3\%B3n\% 202005.pdf (consulta realizada el 09/06/2017).

GARRIDO NOGUERA, C., RONDERO LÓPEZ, N., VEGA MONTOYA, V. (2013) "Innovación, vinculación universidad-empresa y desarrollo. Desafíos y posibilidades de la RedUE en el espacio ALCUE. Unión de Universidades de América Latina y el Caribe", Revista Universidades, vol. LXIII, núm. 58, octubre-diciembre, pp. 6-23, disponible: http://www.redalyc.org/pdf/373/37331247003.pdf (consulta realizada 21/10/2016)

HAUG, G. (2010) "La internacionalización de la educación superior: más allá de la movilidad europea" En: La Cuestión Universitaria, 6, pp. 20-29, disponible en: http://polired.upm.es/index.php/lacuestionuniversitaria/article/view/3392 (consulta realizada el 09/06/2017).

HUERTA, M. (2008) Las redes universitarias: instrumentos para el desarrollo. IAEU, Madrid, España.

KEOHANE, R. (1985) After Hegemony. Cooperation and discord in the world political economy, Princeton University Press, New Jersey, Estados Unidos.

KNIGHT, J. (2005) "An Internationalization Model: Responding to new Realities and Changes". En: De Wit, H.; Jaramillo, I.; Gacel-Ávila, J.; Knight, J.(Eds.) Higher Education in Latin America. The International Dimension (pp. 1-38). Washington, DC : World Bank

(2011) "Is internationalization having an identity crisis?" En: MaldonadoMaldonado, A.; Malee Bassett, R. (eds.) The Forefront of international higher education (pp. 75-87), Springer, Nueva York, Estados Unidos.

LECHINI, G. (2007). "IBSA: una opción de cooperación Sur-Sur", en: Girón, A.; Correa, E. (Comps.), Del Sur hacia el Norte: Economía política del orden económico internacional emergente.. CLACSO, Buenos Aires, Argentina. Disponible: http://bibliotecavirtual.clacso.org.ar/ar/libros/sursur/giron_correa/25Lechini.pdf (consulta realizada 21/10/2016)

MIRANDA, X. (2008) "Integración Regional e internacionalización de la Educación Superior en América Latina y el Caribe. Tendencias y perspectivas. Escenarios en la búsqueda de una decidida complementación", en Gazzora, A. L., Didriksson, A. (Eds.) Tendencias de la Educación Superior en América Latina y el Caribe, UNESCO, Caracas, Venezuela, disponible: http://www.iesalc.unesco.org.ve/index. php?option=com content\&view=article\&id=2\&lte $\underline{\text { mid }=408}$ (consulta realizada 21/10/2016)

OREGIONI, M. S. (2015) "Aspectos sociales de la internacionalización de la investigación. Una propuesta de abordaje”. Revista CTS, V. 10, № 30. Disponible: 
http://www.revistacts.net/volumen-10-numero-30/307-articulos/693-aspectos-socialesde-la-internacionalizacion-de-la-investigacion-una-propuesta-de-abordaje (Consulta realizada 10/06/2017).

PARAMIO, L. (2003): "La globalización y el malestar en la democracia", Documento de Trabajo 02-23, Unidad de Políticas Comparadas (CSIC), Madrid, España, disponible: http://digital.csic.es/bitstream/10261/1545/1/dt-0223.pdf (consulta realizada 21/10/2016)

PERROTA, D. (2016) La internacionalización de la universidad. Debates globales, acciones regionales. Ediciones UNGS- Universidad Nacional de General Sarmiento, Buenos Aires, Argentina.

RAMA, C. (2015) La universidad sin fronteras. La internacionalización de la educación superior en América Latina, Editorial Universitaria de la Universidad Ricardo Palma, Lima, Perú.

RAMOS, D. (2007) "Integración de las redes universitarias: visión y vías de acción desde el IESALC". Revista Gestão Universitária na América Latina - GUAL, vol. 1, núm. 1, 2007, pp. 1-11 Universidade Federal de Santa Catarina, Santa Catarina, Brasil, disponible: http://www.redalyc.org/articulo.oa?id=319327571004 (consulta realizada $21 / 10 / 2016$ )

RICYT (2007) Manual de Santiago. RICYT, disponible: http://www.oei.es/salactsi/manual santiago.pdf (consulta realizada 21/10/2016)

(2015) El Estado de la Ciencia - Principales Indicadores de Ciencia y Tecnología - Iberoamericanos / Interamericanos, RICYT, disponible: http://www.oei.es/salactsi/estado2015.pdf (consulta realizada 21/10/2016)

RUSSELL, R. Y TOKATLIÁN, J. G. (2005) "Argentina, Brasil, EEUU: retos para la cooperación", Revista Papeles de cuestiones internacionales, № 90, verano de 2005 , Pp. 85-96.

SAMPIERI, R. (2010) Metodología de la investigación, 5ta Ed., Mc Graw Hill, México D.F., México.

SÁNCHEZ MARTÍNEZ, E. (2002) La Legislación sobre Educación Superior en Argentina: entre rupturas, continuidades y transformaciones. Entre rupturas, continuidades $y$ transformaciones, IESALC/UNESCO, s/d., disponible: http://unesdoc.unesco.org/images/0013/001398/139827s.pdf (consulta realizada 21/10/2016)

SEBASTIÁN, J. (2004) Cooperación e Internacionalización de las Universidades, Editorial Biblos, Buenos Aires, Argentina

(2013). "La Cooperación Universitaria Iberoamericana: entre la retórica y la incertidumbre", Revista Iberoamericana de Educación № 61 (2013) pp. 45-58 (1022-6508)-OEI/CAEU, disponible en: http://www.rieoei.org/rie61a03.pdf (consulta realizada 01/08/2015) 
Modalidades de cooperación internacional universitaria en el contexto de la internacionalización de la educación superior argentina

Págs. 86-115

SIUFI, G. (2009) "Cooperación Internacional e Internacionalización de la Educación Superior", Educación Superior y Sociedad, Nueva Época, Año 14, № 1, Enero, pp. 119-146, disponible: http://ess.iesalc.unesco.org.ve/index.php/ess/article/viewFile/48/35 (consulta realizada 15/03/2015)

SOCAS, M. y HOURCADE, O. (2009) "La Cooperación Internacional" en: Chiani, A. y Scartascini del Río, J. (Coord.) La cooperación Internacional: herramienta clave para el desarrollo de nuestra región, Buenos Aires, Konrad-Adenauer-Stifung y ACEP, pp.1950, disponible: http://www.kas.de/wf/doc/17526-1442-4-30.pdf (consulta realizada 17/10/2916)

STUBRIN, A. (2011) "Evolución de las políticas universitarias de la argentina durante el período democrático 1983- 2008 y la institucionalización de la evaluación y la acreditación universitaria". En: Albornoz, M. y Sebastián, J. (Eds.) Trayectorias de las políticas científicas y universitarias en Argentina y España, CSIC, Madrid, España.

UNESCO (2009) Comunicado de la Conferencia Mundial sobre la Educación Superior - 2009: La nueva dinámica de la educación superior y la investigación para el cambio social $y$ el desarrollo, autor, París, Francia, disponible: http://www.unesco.org/education/WCHE2009/comunicado es.pdf (consulta realizada el 21/11/2015)

VESSURI, H. (2008) "De la pertinencia social a la sociedad del conocimiento", en: Tünnermann Bernheim, C. (Ed.) La educación superior en América Latina y el Caribe: diez años después de la conferencia mundial de 1998, Pontificia Universidad Javeriana, Santiago de Cali, Colombia.

WITTROCK, B. (1993). "The modern university: The three transformations". En: Rothblatt, S. and Wittrock, B. (Eds.) The European and American University since 1800 (pp. 298-314) Cambridge University Press, Cambridge, Inglaterra.

\begin{tabular}{|c|}
\hline $\begin{array}{l}\text { Michelini Gabriela. Docente investigador de la Universidad Nacional de La Matanza, } \\
\text { Departamento de Humanidades y Ciencias Sociales, Centro de Estudios en Humanidades y } \\
\text { Ciencias Sociales, Co-director del proyecto de investigación PROINCE 55A196 "Características de } \\
\text { la comunicación de la ciencia en la cooperación universitaria". Investigador del Centro de } \\
\text { investigación en Políticas Educativas y Educación Superior, Universidad Austral. Doctora en } \\
\text { Relaciones Internacionales } \\
\text { gabrielaalejandramichelini@gmail.com }\end{array}$ \\
\hline
\end{tabular}


Modalidades de cooperación internacional universitaria en el contexto de la internacionalización de la educación superior argentina Págs. 86-115

Ettlin Mónica Vivian. Docente investigador (en categorización) de la Universidad Nacional de La Matanza, Departamento de Humanidades y Ciencias Sociales, Editor de la revista RIHUMSO (revista de investigación del Departamento mencionado) período oct. 2012-oct. 2016. Doctoranda en Ciencias Jurídicas por la Universidad Católica Argentina, con experiencia profesional en el ámbito del derecho administrativo y en la gestión pública nacional. viviettlin@gmail.com

Luján Acosta Fernando. Decano del Departamento de Humanidades y Ciencias Sociales de la Universidad Nacional de La Matanza, Centro de estudios en Humanidades y Ciencias Sociales, Director del proyecto de investigación PROINCE 55A196 "Características de la comunicación de la ciencia en la cooperación universitaria". Director del Programa de Investigación "Comunicación de la ciencia". 